\title{
Evolution de la politique de prévention des inondations et aménagement de la Loire
}

\author{
par Jean Grassin
}

Chargé de mission risques naturels, Direction de l'aménagement et de l'urbanisme, Ministère de l'Equipement

Un rapide retour sur l'histoire des travaux de protection contre les crues de la vallée de la Loire, constitue la première partie de la communication, utile pour mieux situer la politique de prévention actuelle, qui sera l'objet de la seconde partie.

Quelques considérations générales sur la méthode pour une appropriation par l'ensemble des acteurs de la société de politique de prévention formeront la conclusion.

\section{LES OUVRAGES DE PROTECTION CONTRE LES CRUES DE LA LOIRE AU FIL DES SIÈCLES}

\subsection{L'installation sur les tertres}

La fertilité naturelle des sols de la vallée de la Loire et la proximité de l'eau ont depuis l'antiquité attiré des hommes, qui s'y sont installés pour cultiver la terre. Prudents, ils ont construit leur habitat sur des tertres, formés par des dépôts d'alluvions anciens, hors d'atteinte des plus fortes crues.

A cette époque, où la Loire n'était nulle part endiguée, les plus fortes crues ne s'élevaient jamais à plus de 15 pieds audessus du niveau de l'étiage.

\subsection{Les turcies pour se protéger de l'effet des courants}

Le dépôt de limon fertilisant sur les terres après une inondation est un bienfait de la nature, dont aucun agriculteur de la vallée de la Loire n'aurait voulu se priver.

Par contre lorsque le relief du terrain provoque des zones de courant, la crue devient dévastatrice, elle ravine et emporte les terres arables et laisse après la décrue un sol couvert de sable.

Les paysans, pour s'en défendre, construisent eux-mêmes de petites digues discontinues de faible hauteur placées aux endroits où la rive plus basse est à l'origine des courants de débordement. Ce sont les turcies submersibles, elles ne sont pas très solides mais si une crue les endommage, ce n'est jamais très grave et elles seront facilement réparées.

\section{ㄷ․ㄹ Les turcies médiévales}

Les grands propriétaires terriens qui tirent profit de l'agriculture ont intérêt, pour valoriser leur patrimoine, à construire des digues de protection beaucoup plus importantes que celles qui avaient été construites à l'origine par les paysans.

C'est le début des grands travaux d'endiguement de la Loire. Les nouvelles digues sont censées résister aux plus grandes crues et mettre définitivement les vals à l'abri des inondations. Les premières digues de ce type ont été construites dans la région Angevine à partir de l'année 1160 sous l'égide d'Henri II Plantagenet.

\subsection{L'extension des levées insubmersibles du $\mathrm{Xv}^{\mathrm{e}}$ au} $\mathrm{XIX}^{\mathrm{e}}$ siècle

Par un hasard de l'hydrologie, il n'y a pas eu de très grandes crues de la Loire aux XIII ${ }^{\mathrm{c}}$ et XIV ${ }^{\mathrm{e}}$ siècles jusqu'en 1396, puis de nouveau un siècle s'est écoulé sans grande crue jusqu'en 1494.

Les premiers grands endiguements n'ont ainsi guère été mis à l'épreuve.

L'illusion d'un succès définitif remporté sur la nature a conduit à une erreur fatale: la confiance accordée aux endiguements a fait perdre de vue le danger des très grandes crues.

Des endiguements ont été construits sur de grandes longueurs à partir du $\mathrm{xv}^{\mathrm{e}}$ siècle, réduisant considérablement le champ d'expansion des crues. Le lit majeur naturel large de un à trois kilomètres s'est trouvé réduit, parfois à moins de 500 mètres, sur plusieurs centaines de kilomètres du bec d'Allier jusqu'à Nantes.

Il ne s'agissait plus seulement de protéger des terres agricoles mais bien davantage de fixer le lit du fleuve et ainsi de favoriser la navigation, fort importante pour le commerce et qui faisait la fortune des villes ligériennes.

Les derniers endiguements ont été construits au XIX ${ }^{c}$ siècle pour protéger des terres agricoles dans les vals berrichons et en basse-Loire en aval de l'Authion.

Ces digues dites insubmersibles ont été de nombreuses fois détruites en mains endroits par des crues, par déversement ou par infiltration dans le corps de la levée et phénomène de renard hydraulique. On pensait à chaque fois que la 
dernière crue avait été plus forte que toutes celles que l'on avait connues précédemment, sans se rendre compte que les crues ne pouvant plus s'étaler dans le lit majeur naturel du fleuve, leur débit s'en trouvait renforcé et par voie de conséquence le niveau de la crue plus élevé.

L'histoire garde la trace des crues de 1519, de 1527 et de 1549 au XVI'siècle, de 1608 , de 1628 et de 1649 au $\mathrm{XVII}^{\mathrm{e}}$ siècle, de $1707,1709,1710,1711,1733,1755$ et 1790 au XVIII ${ }^{c}$ siècle. On connaît mieux les trois grandes crues de la Loire du XIX ${ }^{\mathrm{c}}$ siècle qui se sont produites en $1846,1856 \mathrm{et}$ 1866. La dernière crue notable, celle de 1910, n'était que de fréquence cinquantennale et elle n'a pas provoqué de dommages importants.

La hauteur des levées initialement fixée à 15 pieds a été portée ensuite par des plans successifs à 18 pieds, puis à 21 et enfin à 22 pieds en 1707 .

Par deux fois pourtant, en 1629 et en 1711 , le pouvoir royal se rendant compte de l'impossibilité de contenir le débit du fleuve dans son lit endigué trop étroit, avait décidé la construction de déchargeoirs dans les levées pour éviter qu'elles ne soient submergées par les plus fortes crues. Seulement quelques-uns de ces ouvrages ont été construits mais beaucoup d'autres ont été abandonnés en raison des très fortes résistances manifestées par les populations riveraines. Certains déchargeoirs qui avaient été construits ont même été supprimés à partir de 1733 .

Il est également intéressant de se rappeler, que pour faciliter le transport du charbon du bassin de St-Etienne par des chalands naviguant sur la Loire, des travaux de déroctage avaient été réalisés dans les gorges de la Loire pour élargir les passages les plus étroits en amont de Roanne. Ces travaux venaient de s'achever en 1706 lorsqu'une série de crues catastrophiques est arrivée, à partir de 1707. On s'est alors rendu compte de l'impact aggravant de ces travaux et on a décidé de rétablir par un ouvrage le goulot d'étranglement de la Loire. La digue de Pinet construite à cette époque était un barrage submersible en maçonnerie, laissant à la Loire un passage limité à 20 mètres de largeur, dimensionné pour laminer les plus grandes crue dans la plaine du Forez.

Après les trois grandes crus du $\mathrm{XIX}^{\mathrm{e}}$ siècle un nouveau projet de protection contre les crues, reprenant une idée ancienne, a été adopté consistant à construire vingt nouveaux déversoirs dans les levées. Cette fois encore, la résistance des populations riveraines conduira à en abandonner plus de la moítié et finalement sept déversoirs seulement seront construits entre 1870 et 1891 .

\section{L'ÉVOLUTION DE LA POLITIQUE DE PRÉVENTION ET DES PROGRAM- MES D'AMÉNAGEMENT DE LA LOIRE}

L'évolution de la politique de prévention depuis un demisiècle est présentée en suivant la chronologie des modifications de la réglementation. Les grandes étapes des programmes d'aménagement de la Loire sont insérées dans cette chronologie, pour mettre en évidence leur corrélation avec l'évolution réglementaire.

\subsection{Le code de la navigation fluviale}

Jusque dans les années 1950, le développement urbain est resté relativement peu important et le problème de la construction dans les zones inondables ne se posait guère.

La réglementation dans les zones inondables se limitait au décret-loi de 1935 instituant les plans des surfaces submersibles. Ces PSS avaient un objectif purement hydraulique, qui était d'éviter que des constructions, des clôtures ou des remblais ne fassent obstacle au libre écoulement des crues dans les champs d'expansion des cours d'eau.

Dans la vallée de la Loire, des PSS ont été délimités sur l'ensemble du cours de la Loire, à l'exception du département de la Haute-Loire, par des décrets pris à partir de 1958 et jusqu'en 1975.

\subsection{Le code de l'urbanisme}

Le rythme de l'urbanisation s'est brusquement accéléré pendant la période de la reconstruction et de l'industrialisation à partir des années 1950.

Dès 1955, se rendant compte de l'insuffisance de la réglementation des PSS, l'administration chargée de l'urbanisme a introduit dans le code de l'urbanisme l'objectif de la sécurité publique avec l'institution des périmètres de risques (article R 111-3).

Par la suite, diverses modifications du code de l'urbanisme ont rendu obligatoire la prise en compte des risques dans les documents d'urbanisme: les schémas directeurs, les plans d'occupation des sols et les plans des zones d'aménagement concerté

Il faut bien constater cependant que cette prise en compte des risques dans l'aménagement a été notoirement défaillante, en particulier en ce qui concerne les zones inondables, faute d'une volonté politique suffisante des pouvoirs publics à tous les niveaux.

C'est ainsi, par exemple, qu'à Orléans plus de 30000 nouveaux habitants sont venus s'installer, en toute quiétude, dans la zone inondable sur la rive sud de la Loire depuis les années 1960.

\subsection{Un programme de travaux de protection pour la vallée de la Loire}

En 1970 un programme de travaux de protection a été décidé par l'Etat pour réduire le niveau du risque de catastrophes lors du retour attendu de la prochaine crue centennale de la Loire. Ce programme de travaux de protection n'a été accompagné d'aucune mesure de prévention quant au développement de l'urbanisation dans les zones inondables; on peut même considérer qu'il a favorisé l'insouciance.

Ce programme comportait le renforcement de près de 300 kilomètres de levées « insubmersibles " pour éviter le risque de leur rupture par infiltration et la construction de deux barrages écrêteurs de crues pour limiter le débit de pointe des crues centennales à 6000 mètres cubes par seconde à l'aval du bec d'Allier, ce débit correspondant sensiblement à la capacité des endiguements avant débordement.

Le barrage de Villerest situé au débouché des gorges de la Loire en amont de Roanne pour contrôler les crues de la Loire amont a été mis en service en 1984.

Un second ouvrage destiné à contrôler les crues de l'Allier était prévu au Veurdre à une dizaine de kilomètres à l'amont du confluent de I'Allier avec la Loire.

\subsection{L'aménagement intégré de la vallée de la Loire}

En 1979 une association de réflexion pour un aménagement intégré du bassin de la Loire (LIGER) a réalisé une étude d'aménagement portant sur la protection contre les crues, le développement des infrastructures et des activités économiques.

Le ministère de l'Environnement, à qui cette étude avait été adressée, à confié à l'ingénieur général Jean Chapon une mission pour tracer les grandes lignes directrices d'un amé- 
nagement à long terme du bassin de la Loire et proposer la constitution d'une structure politique la mieux adaptée pour être le partenaire de l'Etat pour cet aménagement.

Le rapport Chapon pose le principe de l'aménagement intégré de la vallée de la Loire comme étant la recherche d'un optimum entre la régularisation du fleuve, le développement économique et la protection du patrimoine naturel.

Ce rapport proposait la constitution d'une institution interdépartementale unique pour la maîtrise d'ouvrage des opérations d'intérêt général, notamment celles relative à l'aménagement hydraulique. Cette idée a été à l'origine de la création de l'EPALA, à la fin de l'année 1983.

Pour ce qui est de l'aménagement du territoire, le rapport proposait qu'il soit encadré par des directives d'aménagement émanant de l'Etat et qu'au niveau local des documents de planification intercommunaux soient systématiquement élaborés : schémas directeurs d'urbanisme pour les agglomérations et «plans de val " pour les territoires ruraux.

Plusieurs plans de val ont été lancés mais ils se sont assez rapidement enlisés dans les difficultés de l'intercommunalité et aucun n'a abouti. Rien n'a véritablement changé quant à l'aménagement du territoire ; on en est resté le plus souvent à des vues à court terme et à une échelle territoriale trop petite pour être pertinente. Quant aux schémas directeurs on n'y trouve guère la prise en compte du risque d'inondation.

\subsection{La loi d'indemnisation des catastrophes naturelles et les plans d'exposition aux risques}

Après une série de catastrophes d'inondation le problème de l'indemnisation des dommages s'est trouvé posé et une loi a été votée par le parlement pour instituer un régime d'indemnisation des catastrophes naturelles. Ce régime d'indemnisation est un système hybride de l'assurance de marché et d'un fonds public de solidarité.

Pour éviter une croissance incontrôlée des dépenses d'indemnisation, le législateur a introduit dans la loi un dispositif pour la prévention des risques naturels: les plans d'exposition aux risques. Ces PER avaient pour objet d'interdire l'implantation de nouvelles constructions dans les zones les plus exposées, d'imposer des prescriptions pour imiter la vulnérabilité des constructions nouvelles pouvan être autorisées dans les zones peu exposées et de prescrire des travaux pour réduire la vulnérabilité des constructions existantes.

Treize ans après le vote de cette loi, un peu moins de 400 PER ont été approuvés, c'est un bien maigre bilan.

\subsection{La loi sécurité publique}

La loi du 22 juillet 1987 crée un nouveau droit, le droit des citoyens à l'information sur les risques auxquels ils sont exposés et sur les mesures prises pour assurer leur sécurité.

Il reste encore beaucoup à faire pour que cette information soit réellement disponible et diffusée dans une forme appropriée; la démarche est néanmoins engagée de manière irréversible.

\subsection{L'atlas des zones inondables de la vallée de la Loire}

En 1989 le ministre de l'environnement a chargé le directeur de l'architecture et de l'urbanisme d'une nouvelle réflexion sur la maîtrise de l'espace de la vallée de la Loire.

Parmi les propositions du rapport de Jean Frebault l'accent était mis sur la nécessité d'afficher une position de l'Etat ambitieuse dans la maîtrise des risques, de clarifier et de mieux diffuser la connaissance des phénomènes d'inondabilité en élaborant un atlas des zones inondables.
Par ailleurs, le rapport suggérait la mise en place d'un système d'observation de l'évolution du territoire ligérien.

La publication de l'atlas des zones inondables de la vallée de la Loire, du bec d'Allier jusqu'à Nantes, est aujourd'hui achevée ; c'est un résultat non négligeable.

Cet atlas des zones inondables s'est avéré par son contenu et par sa présentation un très bon outil d'information des élus et du public, qui répond bien au droit des citoyens à l'information sur les risques auxquels ils sont exposés, institué par la loi de juillet 1987.

Dès lors que le risque est connu, rien n'est plus comme avant, le comportement des acteurs de l'aménagement change, l'expérience le prouve.

\subsection{L'affichage des objectifs pour la prévention des inondations}

L'affichage des objectifs et des principes de la politique de l'Etat en matière d'occupation de l'espace dans les zones inondables a fait l'objet de la circulaire interministérielle «Equipement Environnement Intérieur » du 24 janvier 1994.

Cette circulaire initialement conçue pour la seule vallée de la Loire, a finalement vu sa visée élargie à l'ensemble des zones inondables.

Cette circulaire définit un triple objectif :

- un objectif de sécurité des personnes et des biens qui se traduit par l'interdiction des implantations humaines dans les zones les plus dangereuses où, quels que soient les aménagements, la sécurité des personnes ne peut pas être garantie intégralement, et sa limitation dans les autres zones inondables :

- un objectif hydraulique de préservation de capacités d'écoulement et d'expansion des crues pour ne pas aggraver les risques pour les zones situées en amont et en aval,

- un objectif d'environnement, la sauvegarde de l'équilibre des milieux dépendants des petites crues et de la qualité des paysages.

Ces objectifs se traduisent par trois principes :

- l'arrêt de l'extension de l'urbanisation dans les zones inondables pour préserver les zones d'expansion des crues,

- l'interdiction de toute construction nouvelle dans les zones exposées à un risque grave, même à l'intérieur des zones urbaines existantes,

- l'interdiction de tout nouvel endiguement qui n'aurait pas pour objet la protection d'une zone urbaine déjà existante.

Cette circulaire innove car rien d'aussi net n'avait jamais été écrit auparavant sur la position de l'Etat en la matière.

\subsection{Le plan Loire grandeur nature}

Le plan Loire grandeur nature adopté par le gouvernement le 4 janvier 1994 a pour objectif de concilier la sécurité des populations à l'égard des inondations, la satisfaction de leurs besoins en eau et la protection et la mise en valeur de l'environnement.

Nous ne ferons pas une description du contenu de ce programme, nous nous contenterons de constater qu'il s'inscrit dans le droit fil de l'évolution de la politique de prévention. Les grands travaux de protection ne sont plus la priorité, leur utilité publique est à démontrer après comparaison avec d'autres solutions.

C'est ainsi que le projet d'ouvrage écrêteur du Veurdre fait actuellement l'objet d'un moratoire pour permettre de vérifier sa nécessité, compte tenu de l'effet des travaux de nettoyage du lit qui seront réalisés en priorité et après que les mesures de prévention en matière d'urbanisme aient été effectivement prises. 


\subsection{La relance de la prévention des risques}

Janvier 1994, c'est aussi la décision prise par le gouvernement d'un programme national de prévention des risques naturels.

Ce programme relance la politique de prévention par une série d'actions pour une meilleure prise en compte des risques naturels dans les politiques urbaines, et une nouvelle gestion des zones inondables.

Ce programme comporte notamment l'annonce d'une simplification du dispositif juridique de la prévention, la modernisation des réseaux de surveillance et d'annonce des crues, un plan décennal de restauration et d'entretien des rivières.

\subsection{La loi relative au renforcement de la pro- tection de l'environnement}

La loi du 2 février 1995 modifie profondément le dispositif de prévention des risques naturels en instituant un nouvel instrument: le plan de prévention des risques naturels prévisibles, dit PPR.

Le PPR se substitue à tous les documents juridiques antérieurs : les PER, les PSS, les R-111-3 et les plans de zones sensibles aux incendies de forêts. C'est une grande simplification et c'est beaucoup plus clair.

Le PPR est modulable, il peut prendre en compte un seul risque ou plusieurs. Il peut ne comporter dans un premier temps que des mesures relatives aux nouvelles constructions, qui sont les plus urgentes à prendre et ensuite être complété par des mesures applicables aux constructions existantes pour réduire leur vulnérabilité.

Le PPR peut réglementer l'exploitation agricole, forestière, artisanale, commerciale ou industrielle.

Il peut aussi prévoir des mesures de prévention collective : travaux de protection, travaux de nature à faciliter l'évacuation des personnes et des opérations de secours.
La procédure entièrement déconcentrée est simple, le projet de PPR élaboré par les services de l'Etat est soumis à l'avis des conseils municipaux des communes concernées et à une enquête publique, puis éventuellement modifié pour tenir compte de ces avis, approuvé par le préfet.

Le PPR approuvé constitue une servitude d'utilité publique.

\section{III a QUELQUES CONSIDÉRATIONS SUR LA MÉTHODE}

Dans une démocratie l'objectif final de toute politique publique, quelque soit son objet spécifique, c'est ou ce devrait être, un objectif social.

Le rôle de l'Etat devrait être plutôt celui d'un guide qui éclaire l'avenir que celui d'une providence chargée de toutes les responsabilités. Dans cette perspective d'un Etat animateur, sa mission consiste à organiser la société pour qu'elle puisse se mouvoir et tisser des liens entre les différents groupes sociaux pour maintenir sa cohésion.

Dans le domaine de la prévention des risques naturels on voit bien l'importance et l'urgence de diffuser une information pertinente. Si tous disposent de la bonne information, personne, ni un particulier ni un industriel n'aura plus l'idée d'aller s'installer dans une zone très exposée, pour peu que l'on fasse évoluer le système d'indemnisation des dommages pour laisser un peu plus de place à la prévention et la responsabilité.

L'articulation des pouvoirs publics en matière d'aménagement du territoire doit aussi laisser un espace de liberté suffisant au niveau local.

De même qu'il faut laisser libre le champ d'expansion des crues pour éviter les catastrophes, il faut, aussi dans la plus grande transparence, laisser un champ d'application à la responsabilité à tous les niveaux. 